\title{
Efficacy of an improved method to screen semiochemicals of insect
}

\author{
Chongyang Li ${ }^{1}$, Jianmin Cao ${ }^{1}$, Xiufang Wang ${ }^{1}$, Pengjun Xu ${ }^{1}$, Xinwei Wang ${ }^{1}$, Guangwei Ren ${ }^{\text {Corresp. } 1}$ \\ 1 Qingdao Special Crops Research Center, Chinese Academy of Agricultural Sciences, Qingdao, China \\ Corresponding Author: Guangwei Ren \\ Email address: renguangwei@caas.cn
}

Background. A combination of gas chromatography-electroantennographic detection (GC-EAD) and gas chromatography-mass spectrometry (GC-MS) is typically used to screen active compounds that play a role in the regulation of insect behavior. This method uses two gas chromatography (GC) equipments and needs to compare compounds between the two chromatograms, and it is tedious and costly. To improve detection efficiency, as well as reduce costs and the rate of missed detection, we designed a system connecting gas chromatography (GC), electroantennography (EAG), and mass spectrometry (MS), with MS used instead of the flame ionization detector (FID) as the GC-EAD detector. To verify the feasibility of the improved method, we compare two methods-GC-EAG-MS and GC-EADthrough a series of experiments. Some researchers made similar improvements, but these were not compared with GC-EAD, and their method needed to be improved in the synchronization and split ratio. Our method has been optimized and improved in these aspects. Methods. Helicoverpa armigera was the test organism, the improved method and conventional method were used to detect known and unknown compounds, as well as screen out active compounds that could generate responses in $H$. armigera antennae. Results. Screening of known single compounds using two methods, the active compound benzaldehyde was detected in all seven concentrations of solution. By using the two methods, five same active compounds of Helicoverpa armigera were detected in high concentration solution of the mixed compounds ( $\left.100 \mathrm{mg} \mathrm{L}^{-1}, 50 \mathrm{mg} \mathrm{L}^{-1}\right)$; four same active compounds were detected at $20 \mathrm{mg} \mathrm{L}^{-1}$ concentration; two identical same compounds were detected in low concentration solution (concentrations of $10 \mathrm{mg} \mathrm{L}^{-1}$ and below). By using the two methods, six identical active compounds of Helicoverpa armigera were detected in unknown compounds. Conclusion. The improved method was consistent with the conventional method in terms of accuracy and sensitivity. However, compared with the traditional methods, Gas chromatography-electroantennographic-mass spectrometry (GCEAG-MS) saved the cost of a GC equipments and a FID equipment, thereby greatly Peer] reviewing PDF | (2021:02:57918:2:0:NEW 30 Apr 2021) 
lowering the experimental cost. In the experiment, GC-EAG-MS combined the two experimental operations of screening active substances by GC-EAD and identifying active substances by GC-MS into one, which not only reduced the experimental steps, but also avoided the false positive caused by the comparison of the two chromatograms, and it greatly reduced the difficulty level of the overall experimental analysis. GC-EAG-MS is more convenient, efficient, economical, and practical, and could confidently replace traditional methods. With further optimization, it could be widely applied in the study of plant and insect chemical ecology. 


\section{Efficacy of an improved method to screen semiochemicals of} 3 insect

4

5

Chongyang Li, Jianmin Cao, Xiufang Wang, Pengjun Xu, Xinwei Wang, Guangwei Ren*

Qingdao Special Crops Research Center, Chinese Academy of Agricultural Sciences, Qingdao 266101, China

Corresponding Author:

Guangwei Ren

Qingdao Special Crops Research Center, Chinese Academy of Agricultural Sciences, Qingdao, 266101, P.R. China

Email address: renguangwei@caas.cn

\section{Abstract}

Background. A combination of gas chromatography-electroantennographic detection (GC-EAD) and gas chromatography-mass spectrometry (GC-MS) is typically used to screen active compounds that play a role in the regulation of insect behavior. This method uses two gas chromatography (GC) equipments and needs to compare compounds between the two chromatograms, and it is tedious and costly. To improve detection efficiency, as well as reduce costs and the rate of missed detection, we designed a system connecting gas chromatography (GC), electroantennography (EAG), and mass spectrometry (MS), with MS used instead of the flame ionization detector (FID) as the GC-EAD detector. To verify the feasibility of the improved method, we compare two methods - GC-EAG-MS and GC-EAD - through a series of experiments. Some researchers made similar improvements, but these were not compared with GC-EAD, and their method needed to be improved in the synchronization and split ratio. Our method has been optimized and improved in these aspects.

Methods. Helicoverpa armigera was the test organism, the improved method and conventional method were used to detect known and unknown compounds, as well as screen out active compounds that could generate responses in H. armigera antennae.

Results. Screening of known single compounds using two methods, the active compound benzaldehyde was detected in all seven concentrations of solution. By using the two methods, five same active compounds of Helicoverpa armigera were detected in high concentration solution of the mixed compounds (100 mg L-1, 50 $\mathrm{mg} \mathrm{L}^{-1}$ ); four same active compounds were detected at $20 \mathrm{mg} \mathrm{L}^{-1}$ concentration; two identical same compounds were detected in low concentration solution (concentrations of $10 \mathrm{~m} \mathrm{~L} \mathrm{~L}^{-1}$ and below). By using the two methods, six identical active compounds of Helicoverpa armigera were detected in unknown compounds.

Conclusion. The improved method was consistent with the conventional method in terms of accuracy and sensitivity. However, compared with the traditional methods, Gas chromatography- 
41

42

43

44

45

46

47

48

49

\section{0}

51

52

53

54

55

56

57

58

59

60

61

62

63

64

65

66

67

68

69

70

71

72

73

74

75

76

77

electroantennographic-mass spectrometry (GC-EAG-MS) saved the cost of a GC equipments and a FID equipment, thereby greatly lowering the experimental cost. In the experiment, GC-EAG-MS combined the two experimental operations of screening active substances by GC-EAD and identifying active substances by GC-MS into one, which not only reduced the experimental steps, but also avoided the false positive caused by the comparison of the two chromatograms, and it greatly reduced the difficulty level of the overall experimental analysis. GC-EAG-MS is more convenient, efficient, economical, and practical, and could confidently replace traditional methods. With further optimization, it could be widely applied in the study of plant and insect chemical ecology.

\section{Introduction}

Plant volatile compounds and insect pheromones are important substances that convey information to insects and play an irreplaceable role in their foraging, feeding, mating, and reproductive activities. Electroantennography (EAG) (Schneider, 1957) and gas chromatography-electroantennographic detection (GC-EAD) (Moorhouse et al., 1969; Arn, Städler \& Raucher, 1975) are effective methods for studying the recognition and perception of these substances in insects, with GC-EAD being widely used to screen chemical mixtures of plant volatile compounds and insect pheromones (Huang et al., 2014; Johnson et al., 2020; Munro et al., 2020; Pawlowski, Sweeney \& Hillier, 2020 ).

Currently, the following methods are used to study collected chemical mixtures (Huang et al., 2014; Milonas, Anastasaki \& Partsinevelos, 2019; Munro et al., 2020 ; Ortiz-Carreon et al., 2019): Active components that play a role in insect behavior regulation are screened using GCEAD, and the whole samples are analyzed using gas chromatography-mass spectrometry (GC$\mathrm{MS})$. EAD active compounds are identified by GC-MS analysis according to their mass spectra and retention times, in comparison with synthetic or authentic standards. Although this method can identify the composition of information material quickly and accurately, it presented limitations in practical applications. For example, (1) GC-EAD and GC-MS are two separate systems, often using different gas chromatography systems. Due to differences in type, carrier gas, and columns between these two gas chromatography systems, the peak shape, retention time and quantity of the analyzed compounds were varied as well. We have to spend a lot of energy comparing the corresponding compounds in the two chromatograms to prevent incorrect identification results, resulting in a more time-consuming experiment. (2) The application of this method required the combination of GC-EAD and GC-MS, which was tedious and costly. (3) If the sample quantity was too small to support the second injection, it was impossible to carry out analysis and screening. The present study aimed to improve the GC-EAD technology by adding a capillary flow purged splitter in the gas chromatography (GC) structure. The GC column outlet was split between EAG and MS using a capillary flow purged splitter with makeup gas. The 
78 flame ionization detector (FID) was replaced by mass spectrometry (MS). This improved method

79 combined the two experimental operations of GC-EAD and GC-MS in one system, which

80 reduced the cost of one GC equipment and one FID equipment, and avoided comparison of

81 different chromatograms. It reduced the time and operation, and improved the efficiency.

82

83 The GC-EAD method is widely recognized by researchers. We improved this method and carried 84 out comparative experiments between the new method and the original method (GC-EAD) to

85 verify whether the improvement is accurate and feasible. Weissbecker et al. (2004) made similar 86 improvements, and this method was used by Paczkowski et al. (2013). However they did not 87 perform any comparative experiments with the original method (GC-EAD). Therefore, our study 88 is an important supplement to the work of Weissbecker et al. (2004). The method described by 89 Weissbecker et al. (2004) needs to be improved in the synchronization and split ratio, and our 90 study has improved these aspects.

91

92

93

94

95

96

To verify the efficacy of the improved method, we screened active substances in known and unknown detected compounds and compared them with data obtained by conventional methods. In this experiment, the single known compound was the artificially configured benzaldehyde solution. The mixed compounds were a mixture of cis-3-hexen-1-ol, myrcene, linalool, methyl salicylate, and trans- $\beta$-caryophyllene. The tested insects were Helicoverpa armigera, and the unknown compounds were the headspace volatiles of $H$. armigera lures.

In this study, we improved a method of the combined use of GC, EAG and MS. Then we verified

101

102

103

104

105

106

107

108

109

110

111

112

113

114

115 the feasibility of this method through a series of experiments. The results showed that the improved method could obtain same results as the conventional method (GC-EAD) under the same conditions, and the improved method was more convenient and efficient. This paper could provide a reference method for the study of chemical ecology of plants and insects.

\section{Materials \& Methods}

\section{Insects}

H. armigera purchased from Henan Jiyuan Baiyun Industrial Co., Ltd. (China) were fed for several generations under controlled conditions at a 14-h light: 10 -h dark photoperiod, $26 \pm 1{ }^{\circ} \mathrm{C}$, and 55\%-65\% relative humidity (Sun, Huang \& Wang, 2012). Larvae were fed an artificial diet. Pupae were sexed and separated accordingly in cages.

\section{Chemicals}

Benzaldehyde (98.5\%) was purchased from Sinopharm Chemical Reagent Co., Ltd., (Shanghai, China). Myrcene (90\%), trans- $\beta$-caryophyllene (80\%), cis-3-hexen-1-ol (98\%), linalool (98\%), eugenol (99\%), benzyl alcohol (99.5\%), 2-ethyl-p-xylene (98\%), $\alpha$-terpineol (98\%) and cis-11- 
116 hexadecenal (95\%) were purchased from Shanghai Macklin Biochemical Co. Ltd. (China), and

117 methyl salicylate (99.5\%) was purchased from Tianjin Beilian Fine Chemicals Development Co.,

118 Ltd. (China). Hexane was purchased from Merck KGaA (Darmstadt, Germany).

119

120

121

122

123

124

125

126

127

128

129

130

131

132

133

134

135

136

137

138

139

140

141

142

143

144

145

146

147

148

149

150

151

152

153

154

155

To facilitate the experiment, the compounds we selected were common floral substances. It was difficult to generate an antennal reaction when the concentration of most compounds was lower than $1 \mathrm{mg} \mathrm{L}^{-1}$; we therefore set the minimum test concentration of the artificial solution to $1 \mathrm{mg}$ $\mathrm{L}^{-1}$.

The benzaldehyde solution was configured with hexane as solvent and was divided into seven concentration gradients, i.e., $100,50,20,10,5,2$, and $1 \mathrm{mg} \mathrm{L}^{-1}$.

A mixture of cis-3-hexen-1-ol, myrcene, linalool, methyl salicylate, and trans- $\beta$-caryophyllene was prepared using hexane as the solvent, at a concentration gradient similar to the above.

Helicoverpa armigera lures were purchased from Pherobio Technology Co., Ltd. (China).

\section{Collection of volatiles}

Headspace sampling was used to collect volatiles from Helicoverpa armigera lures (Sun, Huang \& Wang, 2012; Wei, Zhu \& Kang, 2006) enclosed in a 5-L gas sampling bag. Volatiles were extracted from the bag using an air sampler at a rate of $200 \mathrm{~mL} \mathrm{~min}^{-1}$ and trapped in a glass tube ( $8 \mathrm{~cm}$ long, with an inner diameter of $6 \mathrm{~mm}$ ) containing $60 \mathrm{mg}$ of 60/80 mesh Tenax TA adsorbent (Alltech Assoc., Deerfield, IL, USA) with frits (Agilent Technologies) at both ends. The collected air was then passed through a freshly activated charcoal filter for purification and re-flowed into the gas sampling bag, to form a cycle. Each collection procedure lasted for $12 \mathrm{~h}$ and was replicated five times. Volatiles were desorbed with $600 \mu \mathrm{L}$ hexane and stored at $-20{ }^{\circ} \mathrm{C}$ for subsequent use.

\section{Gas chromatography-electroantennographic-mass spectrometry analyses}

Gas chromatography, electroantennography, and mass spectrometry were used together, with the following specific improvement and application methods. (Fig. 1)

A capillary flow purged splitter with makeup gas (Agilent G3180B) and pneumatic control module (PCM, Agilent G3471A) were installed in the GC. This system could split the effluent of the GC column in a proper proportion and ensure that the MS was under high vacuum and the EAG was at atmospheric pressure. GC (Agilent 7890B) was equipped with an HP-5ms Ultra Inert column $(30 \mathrm{~m} \times 250 \mu \mathrm{m}$ ID and a film thickness of $0.25 \mu \mathrm{m}$, Agilent Technologies $)$. The system needed to meet the following requirements: the effluent of the GC column was split in a ratio of 1 (MS) to 3 (EAG), and the two shunt substances arrive at their respective detectors at the same time. Therefore the specifications of the columns equipped with MS and EAG were 
156

157

158

159

160

161

162

163

164

165

166

167

168

169

170

171

172

173

174

175

176

177

178

179

180

181

182

183

184

185

186

187

188

189

190

191

192

193

194

different. We used the 2-Way Effluent Splitter Calculator (with Makeup) (Agilent Technologies) to calculate the column specifications installed in MS and EAG. MS (Agilent 7010B) was equipped with a DB5MS column $(2.75 \mathrm{~m} \times 150 \mu \mathrm{m}$ ID, Agilent Technologies $)$, and EAG (Syntech) was equipped with a DB5MS column $(1 \mathrm{~m} \times 200 \mu \mathrm{m} \mathrm{ID}$, Agilent Technologies). This setting could meet the above requirements. The carrier gas was high-purity helium $\left(2.25 \mathrm{~mL} \mathrm{~min}^{-}\right.$ $\left.{ }^{1}\right)$. The oven temperature was programmed as follows: initially set at $50{ }^{\circ} \mathrm{C}$ for $2 \mathrm{~min}$, increased to $260{ }^{\circ} \mathrm{C}$ at a rate of $10{ }^{\circ} \mathrm{C} \mathrm{min}^{-1}$, and maintained for $3 \mathrm{~min}$.

EAG specifications: An antenna of one- to two-day-old adults was cut at the base of the flagellum, and the tip of the terminal segment was removed. Using an electrode gel, the excised antenna was mounted on a microelectrode connected to a micromanipulator (MP-15, Syntech). The stimulus controller unit (CS-55, Syntech) provided a stable airflow by maintaining a flow rate of $20-30 \mathrm{~mL} \mathrm{~min}^{-1}$. The effluent conditioner assembly (TC-02, Syntech) was set to a temperature of $250^{\circ} \mathrm{C}$, to prevent the sample from condensing. Signals were amplified with a USB acquisition controller (IDAC-2, Syntech) and transferred to a computer. Data collection and processing were performed using the GC-EAD 2010 software (Syntech) (Sun, Huang \& Wang, 2012, Zhao, Yan \& Wang, 2006). The compounds that flowed to EAG were to the antenna mixed with a charcoal-filtered and humidified air stream. Mounted antennae were placed in the extended end of a glass tube (inner diameter of $8 \mathrm{~mm}$ ). The mixed solution was detected in female antennae, while volatiles of $H$. armigera lures were detected in that of males. Ten EAG recordings were obtained. A response was considered genuine if it was present in at least seven out of the ten replicates collected (Noushini et al., 2019).

MS specifications: The mass spectrometer was operated with the transfer line set at $250^{\circ} \mathrm{C}$, quadrupole at $150^{\circ} \mathrm{C}$, and ion source at $230^{\circ} \mathrm{C}$. Electron impact ionization was employed, with an electron energy of $70 \mathrm{eV}$. The mass range was set at $25-300 \mathrm{~m} / \mathrm{z}$. The compounds that flowed to MS were identified by comparing their mass spectra with that of the NIST library (Agilent Technologies), and confirmed with authentic reference compounds.

During practical applications, the reaction peak start time of the active compound in EAG was consistent with that detected in MS. Therefore, we only needed to record the starting time of the EAG peak of the active compound, and found the corresponding compound at this time in mass spectrum. The corresponding compound was this active compound, and identified it by MS.

The difference between the starting times of the peak recorded by the two software programs was approximately $0.01-0.03 \mathrm{~min}$; this error margin was caused by manual operation. This system could be used not only to screen and identify active compounds, but also eliminate the use for Capillary Flow Purged Splitter and be used as GC-MS alone. 
195

196

197

198

199

200

201

202

203

204

205

206

207

208

209

210

211

212

213

214

215

216

217

218

219

220

221

222

223

224

225

226

227

228

229

230

231

232

233

A $2 \mu \mathrm{L}$ sample was introduced into the GC column by using GC-EAG-MS. Owing to limitations in the experimental set up, GC-EAD was used in a ratio of 1:1, which was different from GCEAG-MS (1:3). To compare GC-EAD with GC-EAG-MS, we used an injection volume of $3 \mu \mathrm{L}$ GC-EAD, to ensure that the volume of sample reaching the antennae was the same for both methods. This comparison was used to test the accuracy of the experimental results obtained using GC-EAG-MS. To test for the sensitivity of GC-EAG-MS, we used an injection volume of $1 \mu \mathrm{L}$ GC-EAD, to ensure that the volume of sample reaching the detector (MS, FID) was the same for both methods.

\section{Gas chromatography and electroantennographic detection analyses}

EAG specifications: Data collection and processing were performed using the GC-EAD 2014v1.2.5 software (Syntech), and the other specifications were set up as described for the GCEAG-MS system above.

Linked GC-EAD analyses were performed using a Shimadzu 2030 GC instrument equipped with an RTX-5 column $(30 \mathrm{~m} \times 250 \mu \mathrm{m}$ ID, and a film thickness of $0.25 \mu \mathrm{m})$, and nitrogen $(1.4 \mathrm{~mL}$ $\mathrm{min}^{-1}$ ) as carrier gas. Each sample was injected into the GC column in a splitless mode. Injection was performed at $220^{\circ} \mathrm{C}$ and FID at $280{ }^{\circ} \mathrm{C}$. Temperature programs were as per the GC-EAGMS system described above. The effluent was partly to the FID and partly to the antenna mixed with a charcoal-filtered and humidified air stream. Mounted antennae were placed in the extended end of a glass tube (inner diameter of $8 \mathrm{~mm}$ ).

\section{Gas chromatography and mass spectrometry analyses}

The compounds were analyzed using a GC-MS system in traditional methods. GC (Agilent7890B) was equipped with an HP-5ms Ultra Inert column (30 m $\times 250 \mu \mathrm{m}$ ID, film thickness was $0.25 \mu \mathrm{m}$, Agilent Technologies). MS (Agilent 7010B) was equipped with a DB5MS column (2.75 m $\times 150 \mu \mathrm{m}$ ID, Agilent Technologies). The carrier gas was high-purity helium $\left(2.25 \mathrm{~mL} \mathrm{~min}^{-1}\right)$. Temperature programs were as per the GC-EAG-MS system described above. MS specifications were set up as described for the GC-EAG-MS system above

\section{Results}

\section{Screening of known single compounds using two methods}

GC-EAG-MS analyses and identification of benzaldehyde solution

Analyzed EAG data, one compound elicited consistent responses in female $H$. armigera antennae (Fig. 2A). Recorded the starting time of the EAG peak of the active compound, and found the corresponding compound at this time in mass spectrum. It was identified as benzaldehyde by MS, according to their mass spectra and retention time, in comparison with authentic standards. All seven concentrations of the solution produced similar results. 
234 Because this method only needed to determine the compound according to the start time of the

235 EAG reaction peak, and this method does not use FID. Therefore, we only attached the

236 corresponding EAG diagram in the paper (similarly hereinafter).

237

238 GC-EAD and GC-MS analyses and identification of benzaldehyde solution

239 The injection volume for GC-EAD was $3 \mu \mathrm{L}$. In GC analyses with FID, one compound elicited

240 responses in female $H$. armigera antennae (Fig. 2B). The injection volume for GC-MS was $3 \mu \mathrm{L}$.

241 Analyzed and compared the corresponding compounds in the two chromatograms, the EAD

242 active compound was identified as benzaldehyde using GC-MS analysis according to their mass

243 spectra and retention times, in comparison with authentic standards. All seven concentrations of

244 the solution produced the same result.

245

246 The injection volume for GC-EAD was $1 \mu \mathrm{L}$. The target compound benzaldehyde was detected

247 in all seven concentrations of solution.

248

\section{Screening of mixed known compounds using two methods}

250 GC-EAG-MS analyses and identification of the mixed solution

251 Analyzed EAG data, five compounds consistent elicited responses in female H. armigera

252 antennae at $100 \mathrm{mg} \mathrm{L}^{-1}$ and $50 \mathrm{mg} \mathrm{L}^{-1}$ concentrations (Fig. 3A). Recorded the starting time of the

253 EAG peak of the active compounds, and found the corresponding compounds at these times in

254 mass spectrum. These were identified by MS, according to their mass spectra and retention

255 times, in comparison with authentic standards. They were cis-3-hexen-1-ol, myrcene, linalool,

256 methyl salicylate, and eugenol, with eugenol being an impurity and not a target compound.

257

258

259

At $20 \mathrm{mg} \mathrm{L}^{-1}$, the active compounds were myrcene, linalool, methyl salicylate, and eugenol. (Fig.

260

261

262

263

264

265 $4 \mathrm{~A})$

266

At concentrations of $10 \mathrm{mg} \mathrm{L}^{-1}$ and below, the active compounds were linalool and methyl salicylate. (Fig. 5A).

The target compounds were detected in all seven concentrations of the solution. (Table 1)

GC-EAD and GC-MS analyses of the mixed solution

267 GC analyses with FID were performed on the mixed solution, with a GC-EAD injection volume 268 of $3 \mu \mathrm{L}$. The injection volume for GC-MS was $3 \mu \mathrm{L}$. Analyzed and compared the corresponding 269 compounds in the two chromatograms, screened compounds were identified by GC-MS

270 according to their mass spectra and retention times, in comparison with authentic standards. 
272 At $100 \mathrm{mg} \mathrm{L}^{-1}$ and $50 \mathrm{mg} \mathrm{L}^{-1}$, five compounds elicited responses in female $H$. armigera

273 antennae, and were identified as cis-3-hexen-1-ol, myrcene, linalool, methyl salicylate, and

274 eugenol. (Fig. 3B).

275

276 At $20 \mathrm{mg} \mathrm{L}^{-1}$, the active compounds were myrcene, linalool, methyl salicylate, and eugenol. (Fig.

277 4B)

278

279 At concentrations of $10 \mathrm{mg} \mathrm{L}^{-1}$ and below, the active compounds were linalool and methyl

280 salicylate. (Fig. 5B)

281

282

283

The injection volume for GC-EAD was $1 \mu \mathrm{L}$. The target compounds were detected in all seven concentrations of the solution. (Table 1)

284

285

286

\section{Screening of unknown compounds using two methods}

GC-EAG-MS analyses and identification of volatiles from H. armigera lures

Analyzed EAG data, six compounds consistent elicited responses in male $H$. armigera antennae

288 (Fig. 6A). Recorded the starting time of the EAG peak of the active compounds, and found the

289

290

291

292

293

294

295

296

297

298

299

300

301

302

303

304

305

306

307

308

309 corresponding compounds at these times in mass spectrum. These were identified by MS, according to their mass spectra and retention times, in comparison with commercial standards or synthesized samples. They were benzyl alcohol, linalool, 2-ethyl-p-xylene, $\alpha$-terpineol, methyl salicylate, and cis-11-hexadecenal (Gothilf et al., 1978; Piccardi et al., 1977).

\section{GC-EAD and GC-MS analyses and identification of volatiles from $H$. armigera lures} The injection volume for GC-EAD was $3 \mu \mathrm{L}$. In GC analyses with FID, six compounds elicited responses in male $H$. armigera antennae (Fig. 6B). The injection volume for GC-MS was $3 \mu \mathrm{L}$. Analyzed and compared the corresponding compounds in the two chromatograms, screened compounds were identified by GC-MS according to their mass spectra and retention times, in comparison with commercial standards or synthesized samples. They were benzyl alcohol, linalool, 2-ethyl-p-xylene, $\alpha$-terpineol, methyl salicylate, and cis-11-hexadecenal, with cis-11hexadecenal being a known sex pheromone of $H$. armigera.

These active compounds were also detected with an injection volume of $1 \mu \mathrm{L}$.

\section{Discussion}

After a method is improved, it is usually compared with the original method to verify whether the improvement is accurate and feasible. The GC-EAD method has been used for decades, and it is widely recognized by researchers. The present study focuses not only on the improvement of this method but also on its verification and comparison with conventional methods. 
311 Weissbecker et al. (2004) made similar improvements, but they only reported the improved

312

313

314

315

316

317

318

319

320

321

322

323

324

325

326

327

328

329

330

331

332

333

334

335

336

337

338

339

340

341

342

343

344

345

method and the practical application of GC-MS/EAD. They did not perform any comparative experiments with the original method (GC-EAD). We did not know whether we would obtain the same results using GC-EAD and GC-MS/EAD under the same conditions. Therefore, in the present study, we compared the two methods-GC-EAG-MS and GC-EAD by performing single compound, known mixture, and unknown compound analyses to verify the accuracy and feasibility of the improved method. Thus, the present study is an important supplement and improvement to the work of Weissbecker et al. (2004).

The method described by Weissbecker et al. (2004) needs to be improved in its synchronization and split ratio, and the current study has improved these aspects. Weissbecker et al. (2004) mentioned that due to the column and EAD interface, the delay of the EAD signal should amount to $2.5 \mathrm{~s}$ compared to the MS signal. The MS and the EAD signals appear synchronously using the method detailed in our manuscript. In the study conducted by Weissbecker et al. (2004), the effluent of the column was split in a ratio of 1 (MS) to 1 (EAD), but in practical application, we prefer to use split ratios such as 1:2 and 1:3 (MS: EAD) because this allows as many samples as possible to enter the EAG equipment, and makes the EAG react significantly. In the present study, the split ratio in GC-EAG-MS could be switched arbitrarily, which did not affect its synchronization. We only needed to replace the column that leads to EAG. The specification of the replaced column could be calculated simply by entering the split ratio in the splitter calculator. Because we used the splitter with makeup gas (Agilent G3180B), it was very convenient to change the column (i.e., there was no need to empty the MS equipment). The split ratio of the GC-MS/EAD in the studies conducted by Weissbecker et al. (2004) and Paczkowski et al. (2013) was 1:1, although their method may not be able to switch the split ratio arbitrarily. If the split ratio of GC-MS/EAD can be changed, the associated columns will inevitably be changed, which will affect the delay time of the EAD signal as reported by Weissbecker et al. (2004). The delay time will no longer be $2.5 \mathrm{~s}$ and will need to be recalculated. Finally, the EAG component in our GC-EAG-MS system can be turned off such that GC-MS can operate independently, which is a highly convenient feature.

In conclusion, comparative experiments between the new and original methods (GC-EAD), which were not carried out by Weissbecker et al. (2004), are reported in the current study. In addition, the accuracy and feasibility of the combined use of GC, EAG, and MS have been verified. The GC-EAG-MS method described herein is optimized and more advanced than that described by Weissbecker et al. (2004). 
347 The equipments were used for the rapid screening and identification of insect active substances

348

349

350

351

352

353

354

355

356

357

358

359

360

361

362

363

364

365

366

367

368

369

370

371

372

373

374

375

376

377

378

379

380

381

382

383

384

in this paper. Most compounds could not elicite an antennal reaction in the EAG device at the concentration of $1 \mathrm{mg} / \mathrm{L}$, we therefore set the minimum test concentration of the artificial solution to $1 \mathrm{mg} \mathrm{L}^{-1}$. On this basis, we compared and verified between the traditional method and the improved method.

Benzaldehyde presents attractive properties to many noctuid insects (Dötterl, Wolfe \& Jürgens, 2005), and could elicit responses in female H. armigera antennae(Wang et al., 2013). Both methods accurately screened and detected this active compound at a concentration of $1 \mathrm{mg} \mathrm{L}^{-1}$. Therefore, the accuracy and sensitivity of GC-EAG-MS were the same as those of conventional methods in detecting a single compound.

Myrcene, linalool, and methyl salicylate play a role in the foraging, mating, and oviposition of $H$. armigera (Du, 2018; Nebapure, 2020). Cis-3-hexen-1-ol is a common odor of green leaves, and eugenol is a component of insect attractants (Ladd, 1984). These compounds also elicited responses in H. armigera antennae (Chen, Zhang \& Qu, 2010; Li et al., 2015; Nebapure, 2020; Sun et al. 2018; Wang et al., 2013). The results obtained here were consistent with those of previous studies. According to a previous study, trans- $\beta$-caryophyllene played a role in prompting female $H$. armigera to lay eggs (Hartlieb \& Rembold, 1996), and while this compound elicited responses in this species (Cribb et al., 2007; Nebapure, 2020), it did not induce a female response in the present experiment. This could be because the female moths used in this experiment were not mating, and trans- $\beta$-caryophyllene was mainly related to the oviposition of $H$. armigera. Also, the overall concentration of the solution used in this experiment was relatively low, and may not have reached the perceptual concentration to affect H. armigera. This result was consistent with that reported by Chen Xiong 2000 and Sun Jiuguang 2010.

In the mixed solution screening experiment, active compounds were accurately screened by the two methods, with consistent experimental results. Both methods detected the target compounds at the lowest concentration of the test solution, indicating that the accuracy and sensitivity of GC-EAG-MS were the same as those of conventional methods, in the detection of mixed compounds.

At $10 \mathrm{mg} \mathrm{L}^{-1}$ concentration, the active compound eugenol, an impurity, did not generate an EAG response in either method but was detected by GC-EAG-MS. Under similar conditions, eugenol remained undetected by GC-EAD. The results here showed that GC-EAG-MS was superior in sensitivity compared to GC-EAD, in terms of detecting certain compounds. 
385 The two methods used to screen the solution of unknown compounds, produced similar results 386 by detecting the same active compounds. Subsequent behavioral experiments were excluded

387 388

389

390

391

392

393

394

395

396

397

398

399

400

401

402

403

404

405

406

407

408

409

410

411

412

413

414

415

416

417

418

419

420

421

422

423

424 from the objective of this study and were therefore not addressed here.

It was a tedious process to analyze and compare two chromatograms. Such limitations occurred in previous study in our laboratory. The screening of active compounds at the ventral end of female Holotrichia oblita revealed that GC-MS retention time was significantly longer than that of GC-EAD, the amount of compound isolated by GC-MS was more than that of GC-EAD, and their peak shapes were different (Fig. 7). It took us a lot of time for analysis and comparison to accurately identify the active compounds. In this study, a large number of compounds were present in volatiles from $H$. armigera lures. During experimental analyses using conventional methods, differences in the retention time and peak shape of the same compound between GCEAD and GC-MS also made the analysis and comparison of the two chromatograms a tedious process. The GC-EAG-MS method only required identifying the corresponding compound in MS, according to the time when the reaction began to appear in EAG. This eliminated the analysis and comparison step between two different chromatograms and made the whole process efficient, with a narrower margin for error.

To summarize, we used two methods to detect known and unknown compounds. By ensuring that the same sample volume reached the antennae, the experimental results of the two methods were similar, highlighting the accuracy of GC-EAG-MS. Further, ensuring that the same sample volume reached the detectors allowed the identification of target compounds at the lowest concentration of the test solution in both methods, indicating that the sensitivity of GC-EAG-MS was the same as that of conventional methods. The above results showed that the accuracy and sensitivity of the GC-EAG-MS method could be guaranteed. This method was superior to the conventional method in the detection sensitivity of some compounds, effectively reducing the possibility of missed detection. Therefore, this method could be applied to screen substances used by insects to process information.

The use of conventional methods such as GC-EAD and GC-MS to screen insect information substances inevitably used different gas chromatography. The two gas chromatograph types, carrier gases or chromatographic columns were different, so this would generate chromatographic results that were quite different in retention time, peak shape, and number of compounds for the same sample. Therefore, one of the limitations of this type of experiment was to accurately analyze and compare two chromatograms, as well as correctly screen out active substances, without making mistakes in the process. Using the GC-EAG-MS method, the two operations were combined in one system, one gas chromatograph was used throughout the experiment, which eliminated the need to compare two different chromatograms. On the premise of ensuring the accuracy of the experimental results, it not only saved time, but also greatly reduced the difficulty of analysis and the probability of error detection, while improving overall 
425 efficiency. Compared with the conventional method, this method eliminated the need for a GC

426 equipment and a FID equipment, markedly reduced the experimental cost, and is economical and 427 practical.

428

429 Conclusions

430 The accuracy and sensitivity of the GC-EAG-MS method was consistent with that of the 431 conventional method, and could replace the latter for screening active insect substances. Here, 432 this method reduced the injection times and eliminated the need for comparison and analysis 433 between different chromatograms, thereby notably reducing the difficulty level of the 434 experiment. Additionally, it was superior to the conventional method in detecting the sensitivity 435 of some compounds. All these factors improved the efficiency of the experiment and effectively 436 reduced error probability. Experimental costs were greatly lowered with the elimination of one 437 GC equipment and one FID equipment, compared with conventional methods. Therefore, the 438 GC-EAG-MS method is convenient, sensitive, economical, and practical, with wide applications 439 in the study of plant and insect chemical ecology pending further optimization.

440

441

442

443

444 References

445 Arn H, Städler E, Raucher S. 1975. The electroantennographic detector - a selective and 446 sensitive tool in the gas chromatographic analysis of insect pheromones. Zeitschrift für

447 Naturforschung C 30: 722-725.

448

449

450

Chen XL, Zhang JH, Qu HL. 2010. Effects of interaction of Populus simonii volatiles on EAG response of Helicoverpa armigera (Hübner). Journal of Xinjiang Normal University (Natural

451 Sciences Edition) 29: 79-83.

452

453

Cribb BW, Hull CD, Moore CJ, Cunningham JP, Zalucki MP. 2007. Variability in odour

454 reception in the peripheral sensory system of Helicoverpa armigera (Hubner) (Lepidoptera: Noctuidae). Australian Journal of Entomology 46: 1-6.

456

Dötterl S, Wolfe LM, Jürgens A. 2005. Qualitative and quantitative analyses of flower scent in 458 Silene latifolia. Phytochemistry 66: 203-213.

459

460

Du LX. 2018. Functional characterization of the long trichoid sensilla in the antennae of female 461 462

463 Gothilf S, Kehat M, Jacobson M, Galun R. 1978. Sex attractants for male Heliothis armigera 464 (Hdn.). Experientia 34: 853-854. 
465

466

467

468

469

470

471

472

473

474

475

476

477

478

479

480

481

482

483

484

485

486

487

488

489

490

491

492

493

494

495

496

497

498

499

500

501

502

503

504
Hartlieb E, Rembold H. 1996. Behavioral responses of females Helicoverpa armigera

(Lepidoptera:Noctuidae) moths to synthetic pigeon pea (Cajanus cajan L.) kairomone. Journal of Chemical Ecology 22: 821-837.

Huang CH, Li JJ, Zhou L, Yan FM. 2014. EAG and GC-EAD techniques. Chinese Journal of Applied Entomology 51: 579-585.

Ladd TL. 1984. Eugenol-related attractants for the Northern corn root worm (Coleoptera:

Chrysomelidae). Journal of Economic Entomology 77: 339-341.

Li WZ, Wang Q, Li HL, Wang J, LI YY, Guo XR, Yuan GH. 2015. Associative learning of Helicoverpa armigera adults to two key floral odours. Acta Ecologica Sinica 35: 3534-3540.

Milonas, PG, Anastasaki E, Partsinevelos G. 2019. Oviposition-induced volatiles affect electrophysiological and behavioral responses of egg parasitoids. Insects 10, 437: 1-13.

Moorhouse JE, Yeadon R, Beevor PS, Nesbitt BF. 1969. Method for use in studies of insect chemical communication. Nature 223: 1174-1175.

Munro HL, Gandhi KJ, Barnes BF, Montes CR, Nowak JT, Shepherd WP, Villari C, Sullivan BT. 2020. Electrophysiological and behavioral responses Dendroctonus frontalis and D. terebrans (Coleoptera: Curculionidae) to resin odors of host pines (Pinus spp.). Chemoecology 30: 215-231.

Nebapure SM. 2020. Electrophysiological Response of Two Moth Species, Helicoverpa armigera and Maruca vitrata to Host Plant (Cajanus cajan) Volatiles National Academy Science Letters-India 43: 423-426.

Noushini S, Perez J, Park SJ, Holgate D, Jamie I, Jamie J, Taylor P. Rectal gland chemistry, volatile emissions, and antennal responses of Male and Female Banana Fruit Fly, Bactrocera musae. Insects 11, 32: 1-12.

Ortiz-Carreon FR, Rojas JC, Cisneros J, Malo EA. 2019. Herbivore-induced volatiles from maize plants attract Chelonus insularis, an egg-larval parasitoid of the fall armyworm. Journal of Chemical Ecology 45: 326-337.

Paczkowski S, Paczkowska M, Dippel S, Schulze N, Schütz S, Sauerwald T, Weiß A, Bauer M, Gottschald J, Kohl C. 2013. The olfaction of a fire beetle leads to new concepts for early fire warning systems. Sensors and Actuators B: Chemical 183: 273-282.

PeerJ reviewing PDF | (2021:02:57918:2:0:NEW 30 Apr 2021) 
505

506 Pawlowski SP, Sweeney JD, Hillier NK. 2020. Electrophysiological Responses of the Beech

507 Leaf-Mining Weevil, Orchestes fagi, to Seasonally-Variant Volatile Organic Compounds

508 Emitted by American Beech, Fagus grandifolia. Journal of Chemical Ecology 46: 935-946.

509

510 Piccardi P, Capizzi A, Cassani G, Spinelli P, Arsura E, Massardo P. 1977. A sex pheromone 511 component of the Old World bollworm Heliothis armigera. Journal of Insect Physiology 23:

512 1443-1445.

513

514 Schneider D. 1957. Elektrophysiologische untersuchungen von chemo-und mechanorezeptoren

515 der antennae des seidenspinners Bombyx mori L.. Zeitschrift für vergleichende Physiologie 40:

$516 \quad 8-41$.

517

518 Steven D. Johnson SD, Sivechurran J, Doarsamy S, Shuttleworth A. 2020. Dung mimicry: the 519 function of volatile emissions and corolla patterning in fly-pollinated Wurmbea flowers. New

520 Phytologist 228: 1662-1673.

521

533

539

541 Weissbecker B, Holighaus G, Schütz S. 2004. Gas chromatography with mass spectrometric and
522

523

524

525

526

527

528

529

530

531

532

534 Wang GH, Lv BQ, Jin QA, Wen HB, Peng ZQ, Yi KX, Zhang CH. 2013. Effects of the

535 combination of plant volatiles and sex pheromone to electroantennogram responses of

536 Helicoverpa armigera. Chinese Journal of Tropical Crops 34: 1781-1785.

537

538

540

542 electroantennographic detection: analysis of wood odorants by direct coupling of insect olfaction

543 and mass spectrometry. Journal of Chromatography A 1056 (1-2): 209-216.

Sun JG. 2010. Electrophysiological and behavioral response of Helicoverpa assulta and $H$. armigera (Lepidoptera:Noctuidae) to tobacco volatiles. D. Phil. Thesis, Institute of Zoology, Chinese Academy of Sciences.

Sun JG, Huang LQ, Wang CZ. 2012. Electrophysiological and behavioral responses of Helicoverpa assulta (Lepidoptera: Noctuidae) to tobacco volatiles. Arthropod-Plant Interactions 6: $375-384$.

Sun XX, Zhang XG, Li X, Dou YC, Dong WX. 2018. Effects of leaf volatiles from blue gum Eucalyptus globulus on oviposition choices of the cotton bollworm Helicoverpa armigera. Journal of Plant Protection 45: 576-584.

Wei JN, Zhu JW, Kang L. 2006. Volatiles released from bean plants in response to agromyzid flies. Planta 224: 279-287.

544 
545 Xiao C, Du JW, Zhang ZN. 2000. Electroantennogram responses of cotton bollworm

546 (Helicoverpa armigera) to several plant volatiles. Acta Agriculturae Jiangxi 12: 27-31.

547

548 Zhao XC, Yan YH, Wang CZ. 2006. Behavioral and electrophysiological responses of

549 Helicoverpa assulta, $H$. armigera, their $\mathrm{F} 1$ hybrids and backcross progeny to female sex 550 pheromones. Journal of Comparative Physiology A 192: 1037-1047. 
Figure 1

The compostion of GC-EAG-MS.

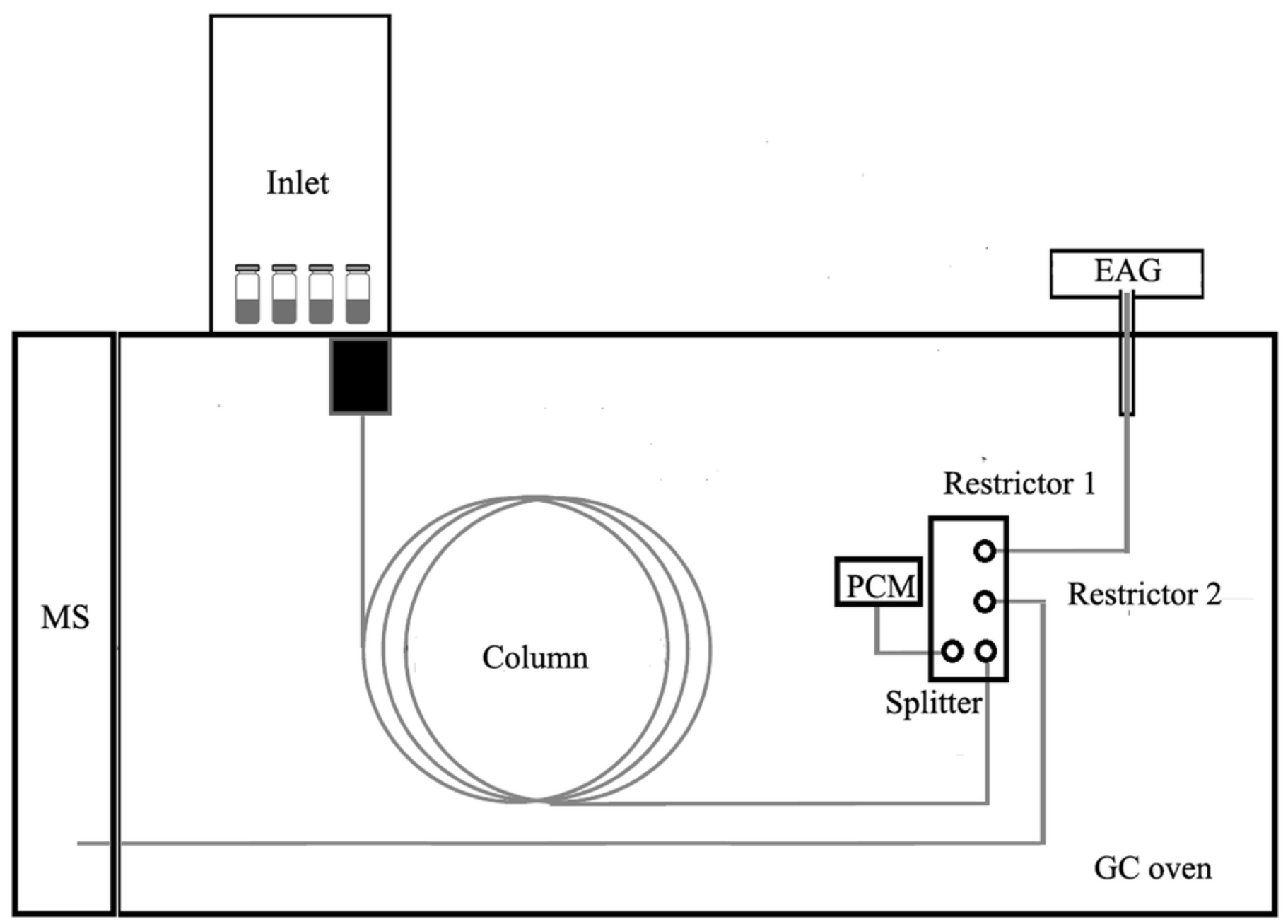


Figure 2

The responses of female Helicoverpa armigera to benzaldehyde solution.

(A) GC-EAG-MS, (B) GC-EAD. The active compound: (1) benzaldehyde.
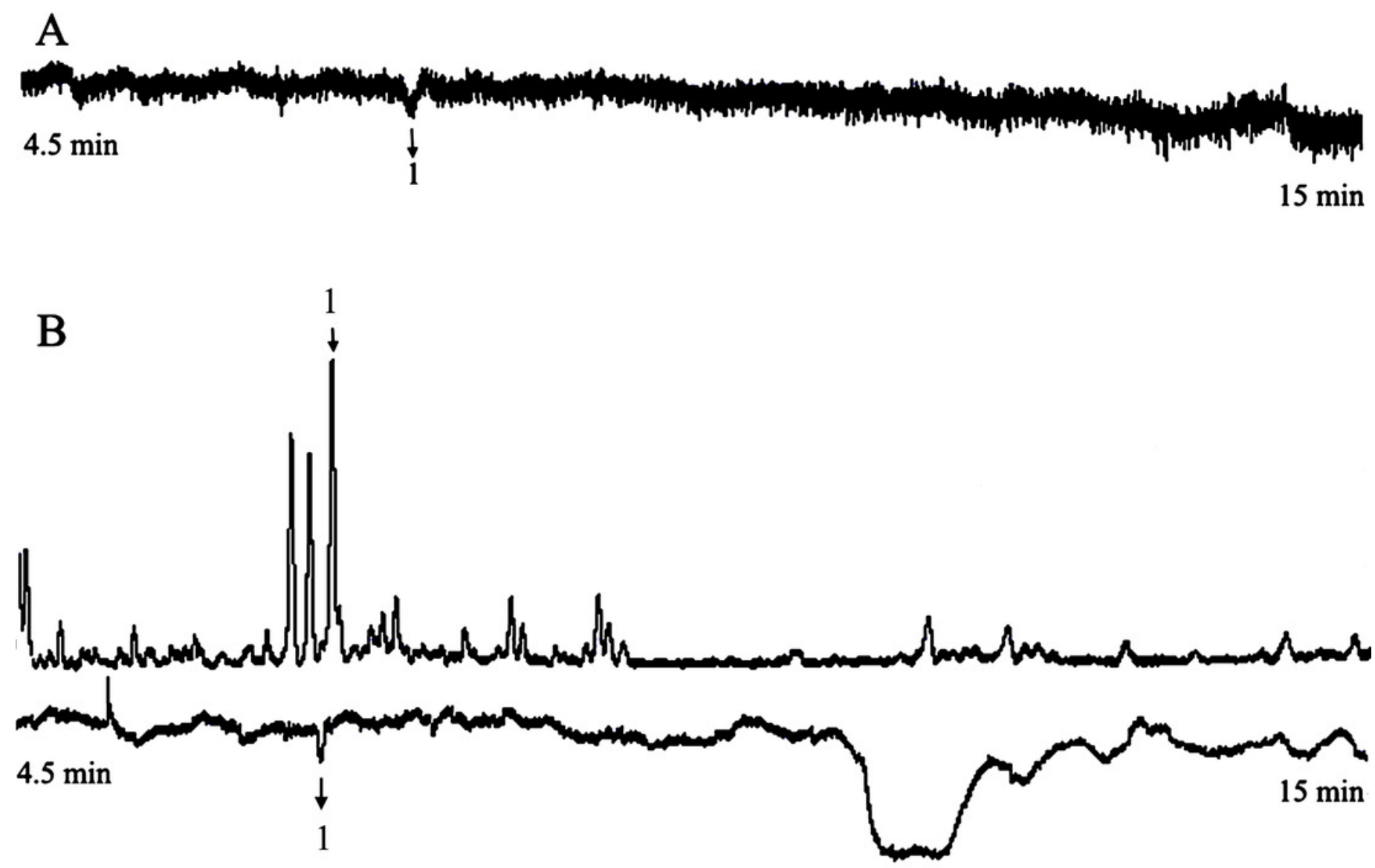
Figure 3

The responses of female Helicoverpa armigera to the mixed solution(50 mg L-1)

(A) GC-EAG-MS, (B) GC-EAD. The active compound: (1) cis-3-hexen-1-ol; (2) myrcene; (3) linalool; (4) methyl salicylate; (5) eugenol. 

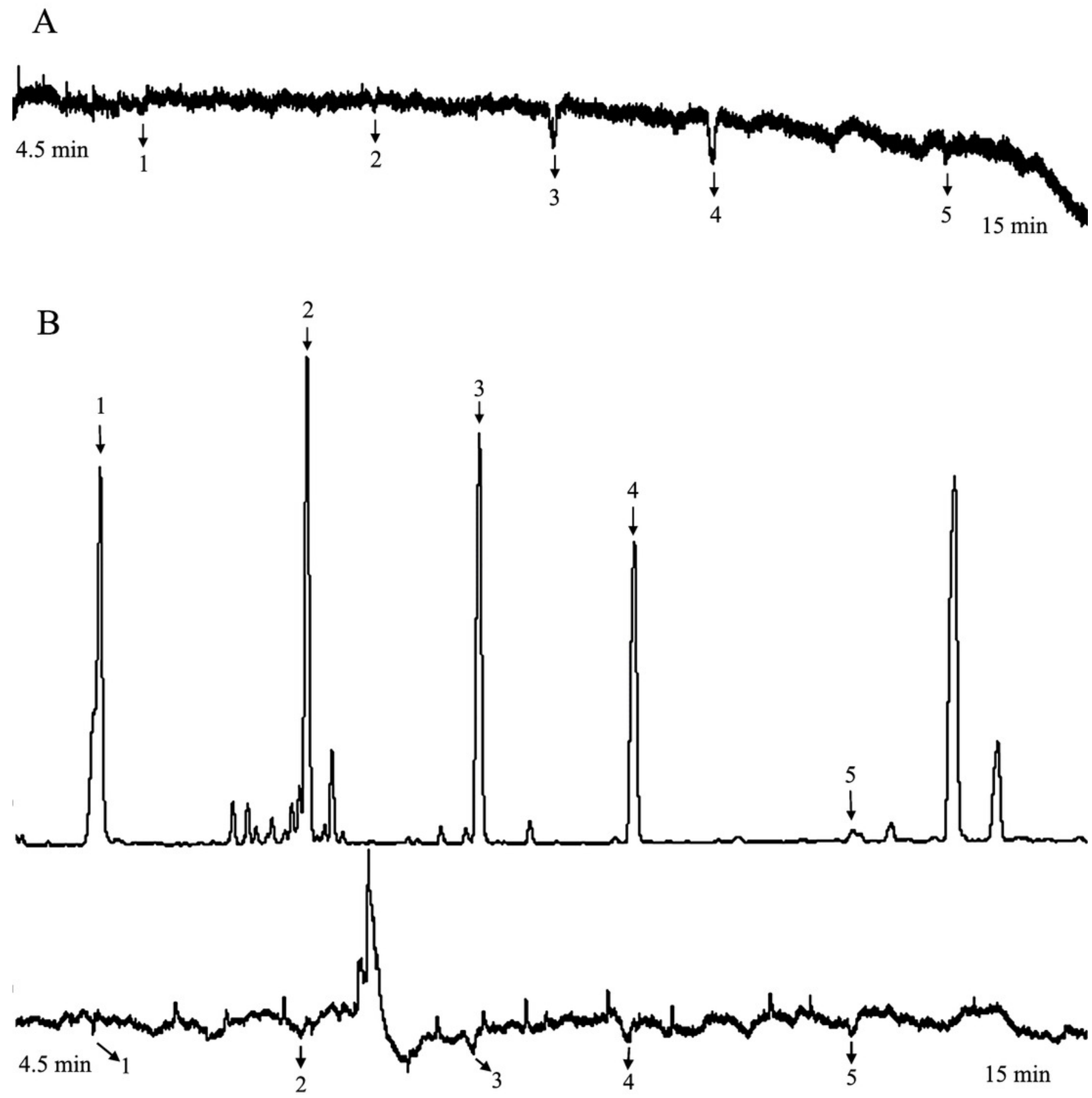
Figure 4

The responses of female Helicoverpa armigera to the mixed solution(20 mg L-1)

(A) GC-EAG-MS, (B) GC-EAD. The active compound: (1) myrcene; (2) linalool; (3) methyl salicylate; (4) eugenol.
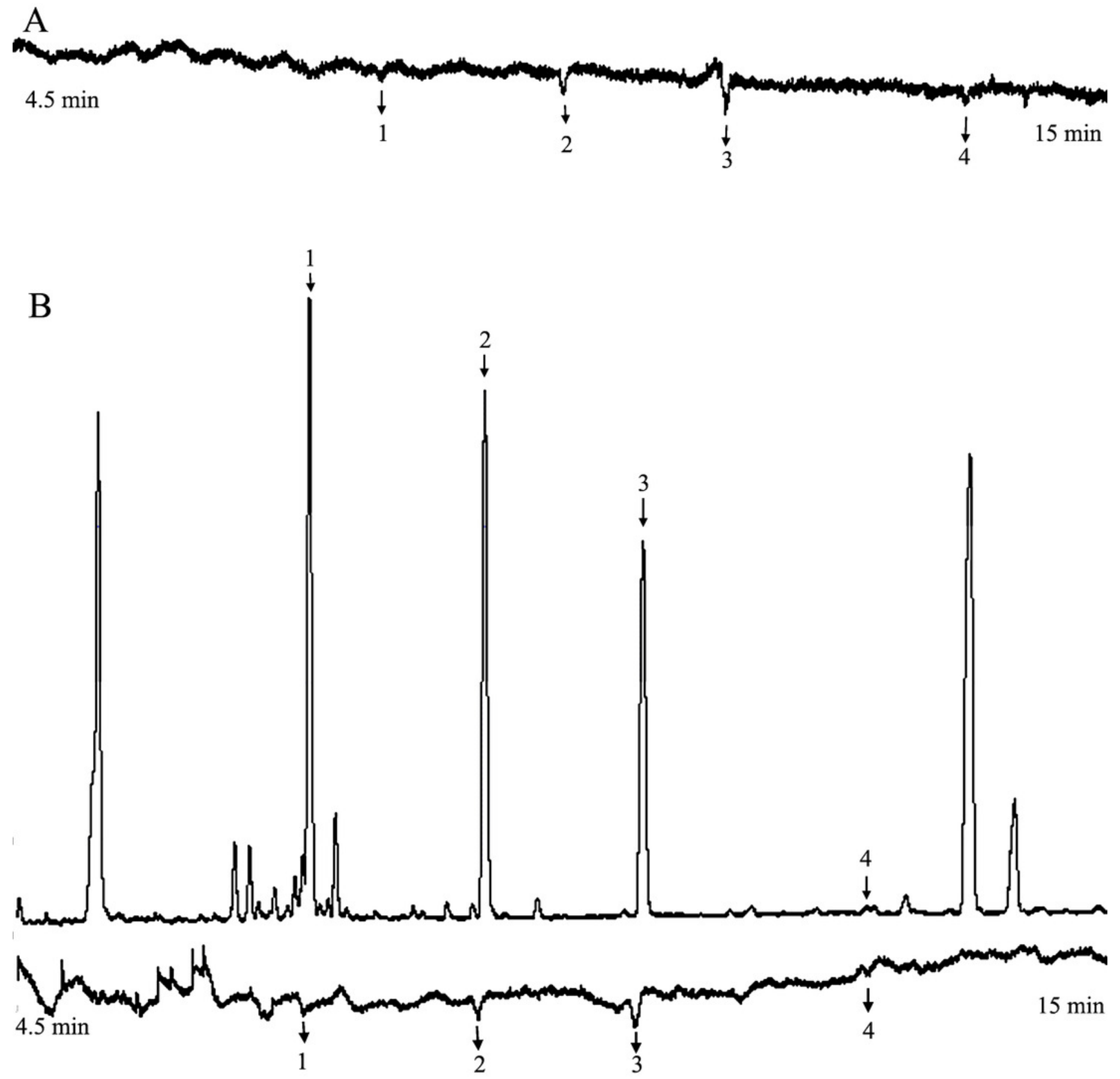
Figure 5

The responses of female Helicoverpa armigera to the mixed solution(1 mg L-1)

(A) GC-EAG-MS, (B) GC-EAD. The active compound: (1) linalool; (2) methyl salicylate.
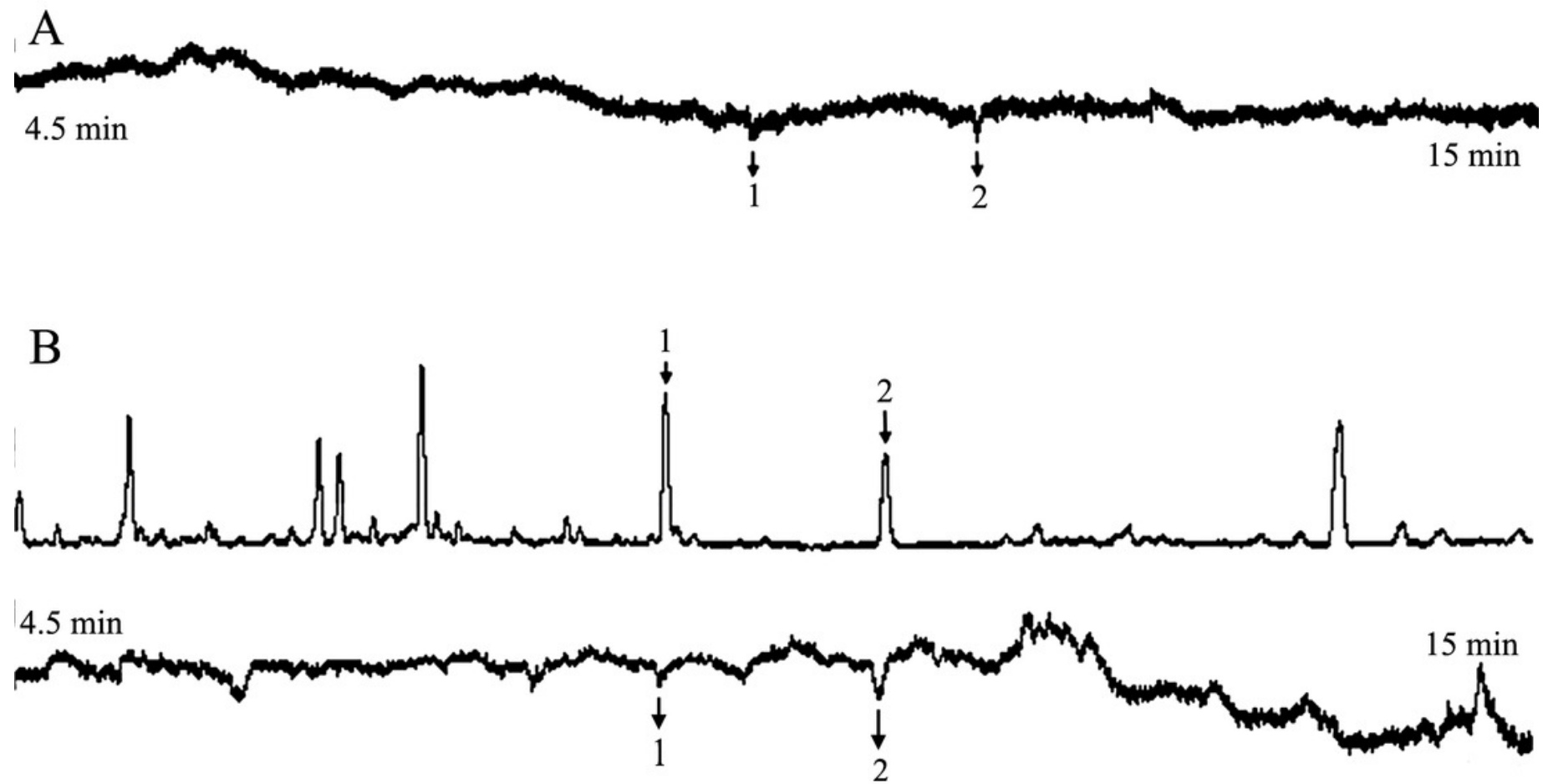
Figure 6

The responses responses of male Helicoverpa armigera to volatiles from the lures

(A) GC-EAG-MS, (B) GC-EAD. The active compound: (1) benzyl alcohol; (2) linalool; (3) 2-ethylp-xylene; (4) $\alpha$-terpineol; (5) methyl salicylate; (6) cis-11-hexadecenal.
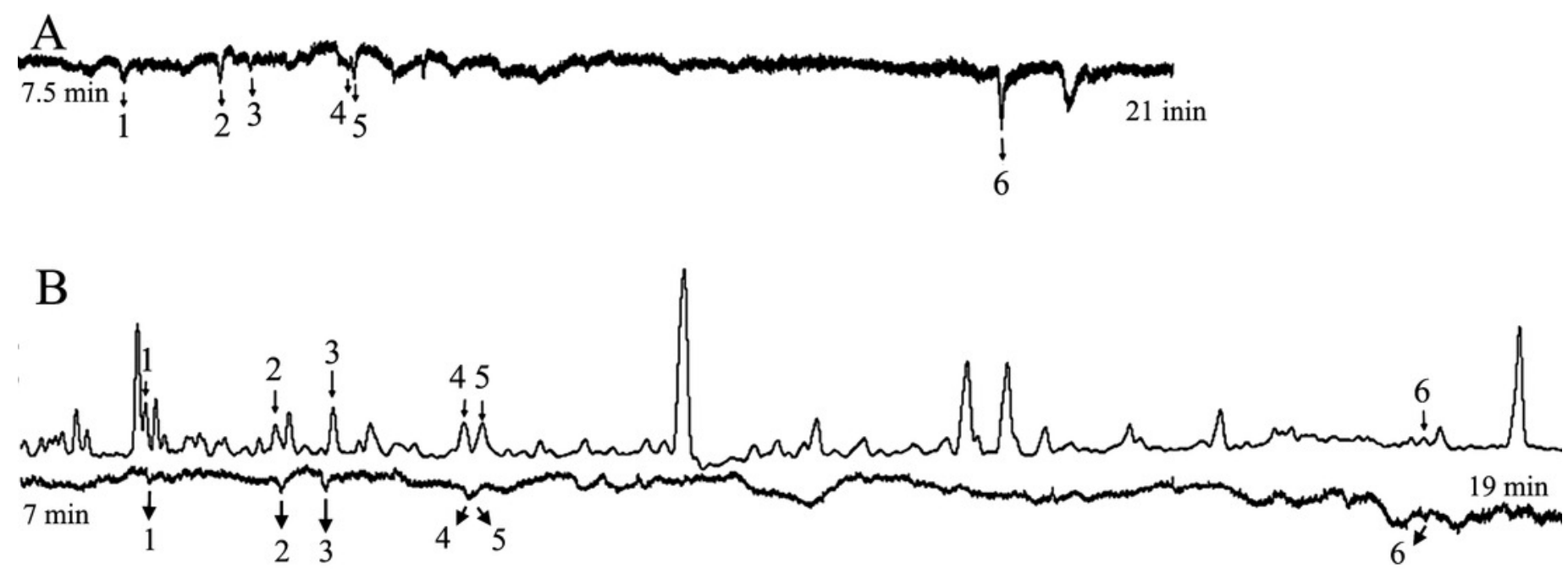
Figure 7

GC-EAD and GC-MS chromatograms of compounds detected at the ventral end of female Holotrichia oblita.
(A) GC-EAD, (B) GC-MS. 


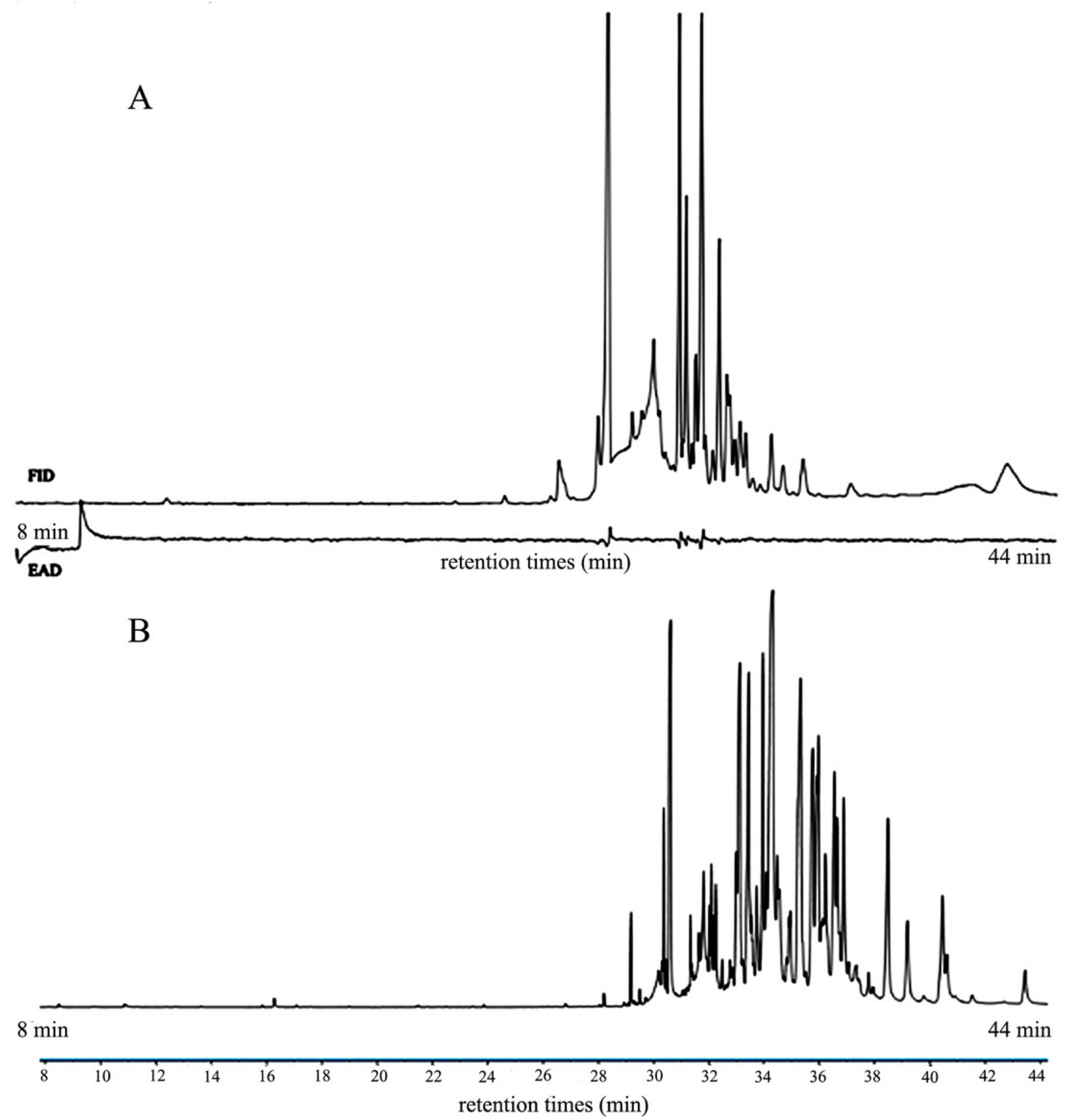




\section{Table 1 (on next page)}

The retention time of the target compounds in the mixed solution using two methods 
1

\begin{tabular}{|c|c|c|}
\hline Method & Compound & Retention time (min) \\
\hline \multirow[t]{5}{*}{ GC-EAG-MS } & Cis-3-hexen-1-ol & 5.82 \\
\hline & Myrcene & 8.06 \\
\hline & Linalool & 9.87 \\
\hline & Methyl salicylate & 11.37 \\
\hline & Trans- $\beta$-caryophyllene & 14.64 \\
\hline \multirow[t]{5}{*}{ GC-EAD } & Cis-3-hexen-1-ol & 5.32 \\
\hline & Myrcene & 7.34 \\
\hline & Linalool & 9.03 \\
\hline & Methyl salicylate & 10.54 \\
\hline & Trans- $\beta$-caryophyllene & 13.68 \\
\hline
\end{tabular}

2 